\title{
Gall bladder wall thickening as non- invasive screening parameter for esophageal varices - a comparative endoscopic - sonographic study
}

Birgit Tsaknakis, Rawan Masri, Ahmad Amanzada, Golo Petzold, Volker Ellenrieder, Albrecht Neesse* and Steffen Kunsch

\begin{abstract}
Background: The mortality due to hemorrhage of esophageal varices (EV) is still high. The predominant cause for EV is liver cirrhosis, which has a high prevalence in Western Europe. Therefore, non-invasive screening markers for the presence of EV are of interest. Here, we aim to investigate whether non-inflammatory gall bladder wall thickening (GBWT) may serve as predictor for the presence of EV in comparison and combination with other non-invasive clinical and laboratory parameters.

Methods: One hundred ninety four patients were retrospectively enrolled in the study. Abdominal ultrasound, upper endoscopy and blood tests were evaluated. GBWT, spleen size and the presence of ascites were evaluated by ultrasound. Platelet count and Child-Pugh-score were also recorded. The study population was categorized in two groups: 122 patients without esophageal varices (non EV) compared to 72 patients with EV were analyzed by uni-and multivariate analysis.

Results: In the EV group 46\% showed a non-inflammatory GBWT of $\geq 4 \mathrm{~mm}$, compared to $12 \%$ in the non-EV group $(p<0.01)$. GBWT was significantly higher in EV patients compared to the non-EV group (mean: $4.4 \mathrm{~mm}$ vs. $2.8 \mathrm{~mm}$, $p<0.0001)$, and multivariate analysis confirmed GBWT as independent predictor for EV $(p<0.04)$. The platelets/GBWT ratio (cut-off $>46.2$ ) had a sensitivity and specificity of 78 and $86 \%$, PPV $76 \%$ and NPV of $87 \%$, and ROC analysis calculated the AUC of 0.864 (Cl 0.809-0.919).
\end{abstract}

Conclusions: GBWT occurs significantly more often in patients with EV. However, because of the low sensitivity, combination with other non-invasive parameters such as platelet count is recommended.

Keywords: Esophageal varices, Gall bladder wall, Cirrhosis, Liver disease, Portal hypertension, Non-invasive parameter, Ultrasound parameter, Child-Pugh-score

\section{Background}

The prevalence of liver cirrhosis is estimated to be between 0.15 and $0.3 \%$ in European countries [1]. The main causes are alcohol abuse, infection with viral hepatitis $\mathrm{B}$ and $\mathrm{C}$ as well as autoimmune liver diseases [2]. A clinically relevant complication is the development of portal hypertension with all its clinical consequences

\footnotetext{
*Correspondence: albrecht.neesse@med.uni-goettingen.de

Department Gastroenterology and Gastrointestinal Oncology, University Medical Centre Goettingen Georg-August-University, Robert-Koch-Str. 40, 37075 Goettingen, Germany
}

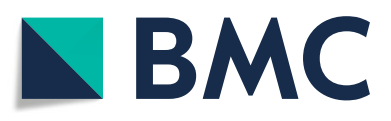

(c) The Author(s). 2018 Open Access This article is distributed under the terms of the Creative Commons Attribution 4.0 International License (http://creativecommons.org/licenses/by/4.0/), which permits unrestricted use, distribution, and reproduction in any medium, provided you give appropriate credit to the original author(s) and the source, provide a link to the Creative Commons license, and indicate if changes were made. The Creative Commons Public Domain Dedication waiver (http://creativecommons.org/publicdomain/zero/1.0/) applies to the data made available in this article, unless otherwise stated.

such as ascites, spontaneous bacterial peritonitis and development of portosystemic collaterals. A progression rate of $12 \%$ has been reported for esophageal varices (EV) [3]. Although the mortality of variceal hemorrhage has declined in the last decades, it is still very high with a six-week-mortality of up to $37 \%$ [4], and a high recurrence rate after the first bleeding incident [5]. Although repeated endoscopic controls of patients with an advanced liver fibrosis or liver cirrhosis are justified, it is an invasive diagnostic procedure with its own risks, and it is not always widely available in countries with lower 
health care standards. Therefore, non-invasive predictors for portosystemic collaterals are of high interest. Notably, the venous blood is drained from the gall bladder in part via small vessels directly into the liver. An additional venous blood drain flows via small veins towards the cystic duct and then with vessels from the common bile duct terminating in the portal venous system [6].Therefore, the gall bladder should be directly affected by portal hypertension causing a thickened gall bladder wall due to impaired venous drainage. Here, we aim to determine whether non-inflammatory gall bladder wall thickness (GBWT) correlates with the presence of EV. To this end, we performed a retrospective endoscopic-ultrasonographic study correlating the presence of EV and GBWT with other non-invasive parameters for liver disease and portal hypertension.

\section{Methods}

In this study we retrospectively included all patients with chronic hepatic disease, who received an ultrasound of the abdomen either as an inpatient or outpatient in the Department of Gastroenterology and Gastrointestinal Oncology of the University Hospital of Goettingen between April 2015 and January 2016. Patients who had a cholecystectomy or complained of upper abdominal pain were excluded from the study. Gall stones and single gall bladder polyps without symptoms were no exclusion criteria. Of all patients who also had a documented upper endoscopy (median time interval 147 days), the following parameters were evaluated by ultrasound: The thickness of the gall bladder was measured twice after overnight fasting at two different locations and an average value was calculated. The spleen length was measured from a left lateral cross section. The diameter of the portal vein, the portal blood flow velocity and the liver size were measured. Ultrasound and endoscopy examinations were performed by experienced Gastroenterology trainees ( $>3$ years experience) and senior Gastroenterology consultants. The presence or absence of ascites was recorded. Additionally, clinical parameters such as the Child-Pugh-classification, laboratory results and upper endoscopic findings (presence of EV graded according the classification of Paquet) were obtained. Using the results of the cranio-caudal spleen diameter, gall bladder wall diameter and laboratory results, we calculated the ratio of platelet count to spleen diameter and the ratio of platelet count to gall bladder wall thickness. The statistical analysis was performed using the Mann Whitney $U$ and Chi square test. Furthermore, variables with a $P$ value $<0.1$ from univariate analysis entered the multivariate binary logistic regression analysis and (receiver operating characteristic) ROC analysis was performed by SPSS Version 25 Mac OS. Since patient data were collected retrospectively and did not influence the diagnostic or therapeutic management of the patients, the ethic committee at the University Medical Centre Goettingen, Germany, was informed in written form about the study prior to data collection but did not request a separate ethical votum $(24 / 7 / 15 \mathrm{AN})$.

\section{Results \\ Patient characteristics}

A total of 194 patients were included in this study, of whom 84 were female and 110 were male. The average age of the patients at time of ultrasound examination was 57 years (range: 17-85 years). The main cause of hepatic disease was alcohol abuse $(n=51)$, followed by unknown cause of liver illness $(n=38)$ and viral hepatitis $\mathrm{B}$ and $\mathrm{C}(n=35)$. The underlying liver diseases are summarized in Fig. 1. The patients were divided into two groups: 122 patients without EV (referred to as "non-EV" group) and 72 patients with EV in endoscopic diagnostic examination (referred to as "EV" group). Of those with EV 31 patients had $1^{\circ}$ varices, 32 patients had $2^{\circ}$ and further 9 patients had $3^{\circ}$ varices. Interestingly, male patients were significantly more often represented in the EV group (73.6\% EV group vs. $46.7 \%$ non-EV group; $p<0.001$ ), potentially reflecting the high percentage $(46 \%)$ of patients with alcohol abuse in the EV group. Histology of the liver was available in $53 \%$ of all patients $(n=102)$, and in those, cirrhosis was confirmed in $63 \%(n=22)$ in the EV-group, and 19\% $(n=13)$ in the non-EV group. As expected, hypertensive gastropathy, advanced Child Pugh Score and presence of ascites occurred significantly more frequently in the EV group. The patient characteristics disease severities are summarized in Table 1.

\section{Ultrasonographic findings}

In the EV group $46 \%$ of patients showed a non-inflammatory (absence of clinical and laboratory signs of acute cholecystitis) GBWT of $\geq 4 \mathrm{~mm}$, compared to $12 \%$ in non-EV group $(p<0.01)$. GBWT was significantly higher in EV patients compared to the non-EV group (mean: $4.4 \mathrm{~mm}$ vs. $2.8 \mathrm{~mm}, p<0.0001$ ) Fig. 2a, b. The median of non-EV was lower with $2.5 \mathrm{~mm}$ than the median of $3.8 \mathrm{~mm}$ in the EV group. A more detailed analysis of the EV group revealed that there was no significant difference between first, second and third degree EV subgroups with an average thickness of $4.3 \mathrm{~mm}, 4.5 \mathrm{~mm}$ and $4.2 \mathrm{~mm}$, respectively.

The spleen size as additional ultrasound parameter is also indicative for portal hypertension. In our cohort, the average spleen length was significantly higher in the EV group compared to the non-EV-group (138 $\mathrm{mm}$ vs. $113 \mathrm{~mm} ; \mathrm{p}<0,001$; Table 2). The portal vein diameter was also significantly higher in the EV group $(12.4 \mathrm{~mm}$ vs. $11.6 \mathrm{~mm} ; p=0.045$; Table 2). Further parameters 


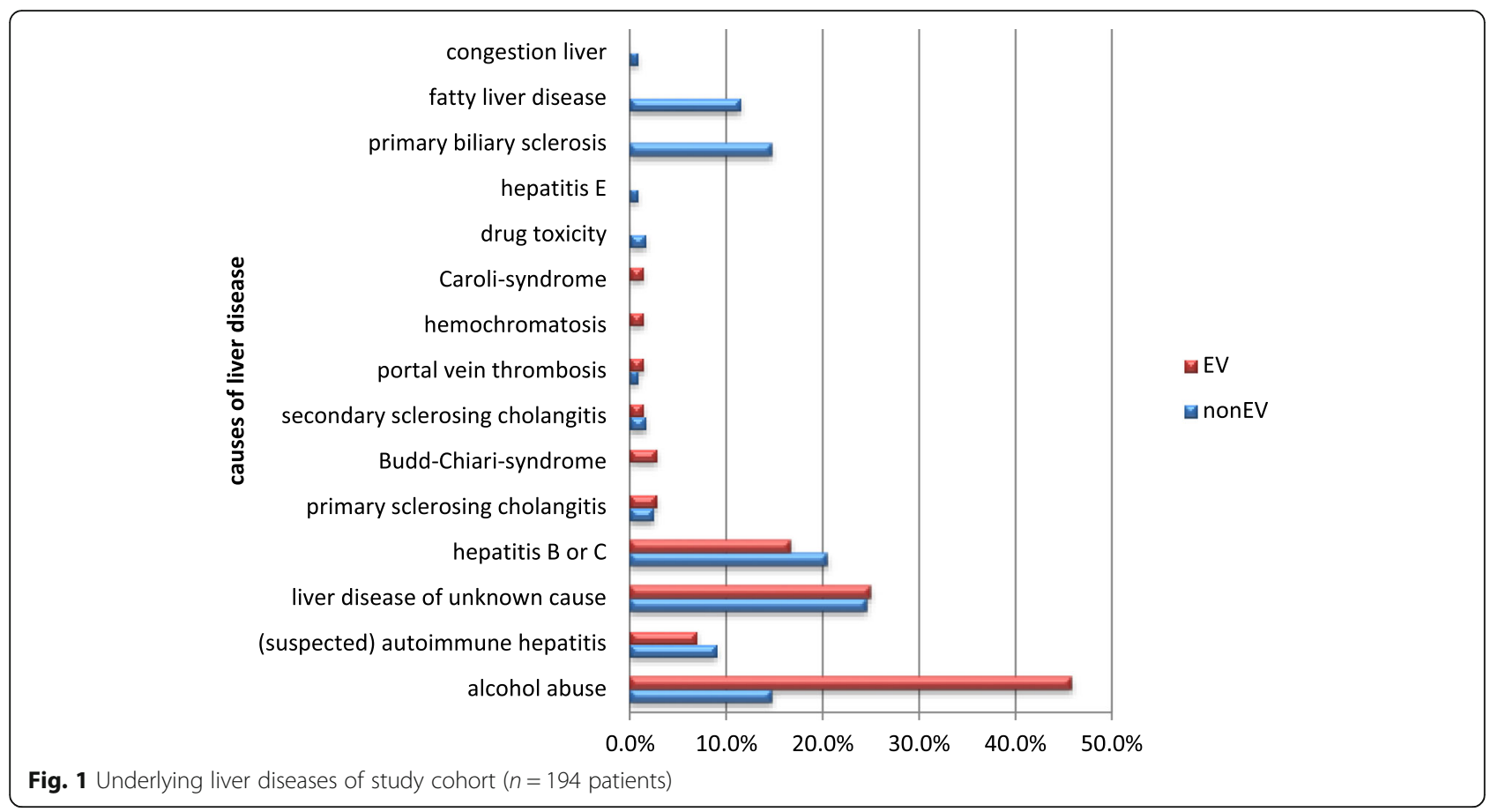

measured by ultrasound such as average portal vein blood flow velocity, gall bladder length, and gall bladder diameter did not show any significant difference between the two groups (Table 2).

\section{Biochemical analysis}

The platelet count was significantly lower in the EV group $(128.000 / \mu \mathrm{l}$ EV group vs. $227.000 / \mu \mathrm{l}$ non-EV group; $p$-value $<0.001$; Table 2).

The average value of the INR differed significantly between the two groups with $1.09 \pm 0.38$ in the non-EV group and $1.39 \pm 0.46$ in the EV group (p-value $<0.0001$ ). Sensitivity, specificity, positive and negative predictive value were calculated for single parameters regarding the presence of $\mathrm{EV}$ and showed sensitivities ranging between 40 and $70 \%$ (Table 3). In particular, the sensitivity of GBWT of $\geq 4 \mathrm{~mm}$ for the presence of $\mathrm{EV}$ was $46 \%$, while

Table 1 Patient characteristics (comparison of group non-EV without esophageal varices and group EV with esophageal varices found by upper endoscopy)

\begin{tabular}{llll}
\hline Parameter & non-EV $n=120$ & $\mathrm{EV} n=72$ & $p$-value \\
\hline Male sex & $47 \%$ & $74 \%$ & $<0.001$ \\
Age (mean) & $57 \pm 14$ & $57 \pm 13$ & $\mathrm{~ns}$ \\
Hypertensive gastropathy & $14 \%$ & $74 \%$ & $<0.0001$ \\
Child-Pugh-Classification A & $92 \%$ & $50 \%$ & $<0.0001$ \\
Child-Pugh-Classification B & $3 \%$ & $26 \%$ & $<0.0001$ \\
Child-Pugh-Classification C & $5 \%$ & $23 \%$ & $<0.001$ \\
presence of ascites & $6 \%$ & $44 \%$ & $<0.0001$ \\
\hline
\end{tabular}

the specificity was $89 \%$. The positive predictive value was $70 \%$ and the negative predictive value $73 \%$.

Using multivariate analysis by logistical regression including sex, Child-Pugh score, GBWT, liver size, spleen diameter, International Normalized Ratio (INR), platelet count, ascites and portal vein diameter, we show that GBWT, ascites, platelet count and spleen diameter are independent predictors of EV (Table 4).

However, platelet count/GBWT ratio (cut-off > 46.2) achieves a sensitivity of $78 \%$ and a specificity of $86 \%$. The positive predictive value is $76 \%$ and the negative predictive value $87 \%$ (Table 5 ). Using our dataset, ROC analysis showed that the platelet count/GBWT ratio performed at a comparable level (area under the curve (AUC) 0.864 (confidence interval (CI) 0.809-0.919)) to the platelet count/spleen diameter ratio of 909 (AUC 0.841 (CI 0.782-0.901)) that was reported by Giannini et al. [7] (Fig. 3).

\section{Discussion}

Patients with compensated liver cirrhosis have a chance of up to $40 \%$ to develop EV [8]. To avoid hemorrhage from $E V$, it is recommended to perform an upper endoscopy as soon as there are signs for the presence of liver cirrhosis in patients $[9,10]$. Therefore, many patients undergo upper endoscopy although they do not require treatment of EV (e.g. ligation) according to endoscopic classifications. While diagnostic gastroscopy itself is of low risk, low platelet counts as well as impaired coagulation parameters increase the risk of complications. Most patients prefer sedation during the procedure which is 
A

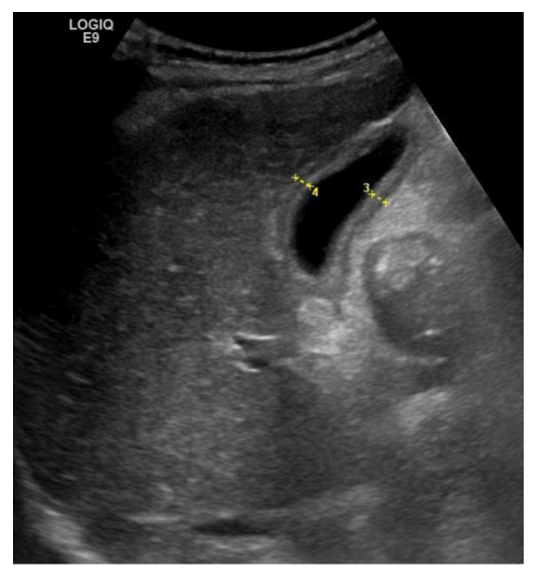

B

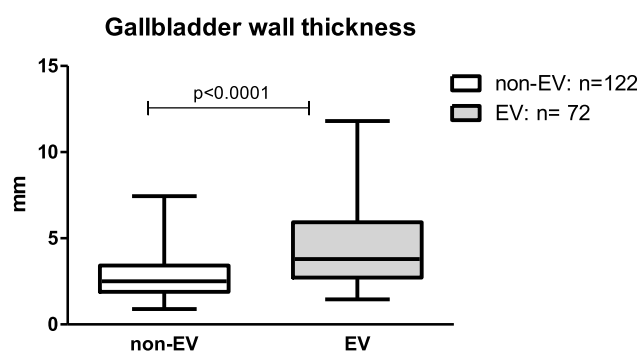

Fig. 2 a Sonographic measurement of gall bladder wall thickness (GBWT) at two different locations. b GBWT is significantly different in patients with esophageal varices (EV) compared to non-EV patients ( $p<0.0001$, Mann Whitney-U test)

associated with additional risks. Therefore, more accurate non-invasive parameters for the presence of EV could be a valuable and clinically relevant tool. We based our study on non-invasive, standard diagnostic tests, which are routinely performed in patients with chronic liver disease: ultrasound, clinical and laboratory results were evaluated in terms of prediction of EV.

Because of its portal-venous blood supply, we assumed that the GBWT may predict the presence of portal hypertension and EV. An interesting study by Maruyama et al. also reported a lower sensitivity of $62 \%$ regarding the detection of large esophageal varices using the platelet count to spleen diameter ratio in 229 cirrhosis patients. In this study, the authors showed that a diameter of the left gastric vein -as a non-variceal collateral- of more than $5.35 \mathrm{~mm}$ had a sensitivity of $90 \%$ and a specificity of $62 \%$ for presence of large esophageal varices. Its sonographic detection was associated with a sensitivity of $84 \%$ for any esophageal varices and a sensitivity of
$100 \%$ for large varices [11]. However, further prospective studies are required to assess the value of portal vein velocity as non-invasive parameter for the presence of esophageal varices.

A small Chinese study showed a correlation between portal vein velocity and GBWT supporting the hypothesis that GBWT could also predict the presence of EV [12]. From a pathophysiological point of view, GBWT may be a microcirculatory driven event caused by impaired portalvenous outflow before significant changes in portal vein velocity occur. However, the development of GBWT may also be caused by other factors such as the serum-ascites albumin gradient (SAAG) [13].

Several studies have investigated non-invasive parameters as predictors for the presence of EV. A platelet count to spleen diameter ratio of 909 and less was associated with EV [7]. The enlarged spleen is caused by portal hypertension and low platelets were also associated with a lowered thrombopoetin serum level due to reduced liver

Table 2 Sonographic, clinical and laboratory findings in patients without esophageal varices (non-EV=122) and endoscopically confirmed varices $(E V=72)$

\begin{tabular}{llll}
\hline Parameter & non-EV $n=120$ & EV $n=72$ & $p$-value \\
\hline Gall bladder wall thickness & $2.8 \pm 1.2 \mathrm{~mm}$ & $4.4 \pm 2.1 \mathrm{~mm}$ & $<\mathbf{0 . 0 0 0 1}$ \\
Gall bladder length & $61.6 \pm 17.6 \mathrm{~mm}$ & $61.1 \pm 21.8 \mathrm{~mm}$ & 0.867 \\
Gall bladder diameter & $25.0 \pm 8.6 \mathrm{~mm}$ & $27.1 \pm 10.0 \mathrm{~mm}$ & 0.128 \\
Liver size in MCL & $13.8 \pm 2.1 \mathrm{~cm}$ & $14.7 \pm 2.6 \mathrm{~cm}$ & $\mathbf{0 . 0 1 5}$ \\
Spleen diameter & $112.9 \pm 23.9 \mathrm{~mm}$ & $138.0 \pm 28.2 \mathrm{~mm}$ & $\mathbf{< . 0 0 0 1}$ \\
Portal vein diameter & $11.6 \pm 2.1 \mathrm{~mm}$ & $12.4 \pm 2.9 \mathrm{~mm}$ & $\mathbf{0 . 0 4 5}$ \\
Portal vein velocity & $18.0 \pm 3.9 \mathrm{~cm} / \mathrm{s}$ & $18.0 \pm 5.8 \mathrm{~cm} / \mathrm{s}$ & 0.968 \\
INR & $1.09 \pm 0.38$ & $1.39 \pm 0.46$ & $<\mathbf{0 . 0 0 0 1}$ \\
Platelet count & $226.6 \pm 85.9 \times 1.000 / \mu \mathrm{l}$ & $128.1 \pm 99.2 \times 1.000 / \mu l$ & $<\mathbf{0 . 0 0 0 1}$ \\
\hline
\end{tabular}

INR International ratio; MCL Medioclavicular line

Significant are all values $<0.05$ 
Table 3 Sensitivity, specificity, positive and negative predictive value for the presence of esophageal varices using gall bladder wall thickness (GBWT) $\geq 4 \mathrm{~mm}$, spleen length ( $\geq 130 \mathrm{~mm}$ ), ascites, thrombocytes $(<160.000 / \mu \mathrm{l})$ and Child-Pugh classification

\begin{tabular}{llllll}
\hline Parameter & GBWT $(\geq 4 \mathrm{~mm})$ & Spleen $(\geq 130 \mathrm{~mm})$ & Thrombocytes $(<160.000 / \mu \mathrm{l})$ & Ascites & Child-Pugh B or C \\
\hline Sensitivity & $46 \%$ & $62 \%$ & $69 \%$ & $44 \%$ & $50 \%$ \\
Specificity & $89 \%$ & $81 \%$ & $78 \%$ & $94 \%$ & $92 \%$ \\
Positive predictive value & $70 \%$ & $67 \%$ & $64 \%$ & $82 \%$ & $78 \%$ \\
Negative predictive value & $73 \%$ & $78 \%$ & $81 \%$ & $74 \%$ & $76 \%$ \\
\hline
\end{tabular}

function [14]. Chen performed a meta-analysis to confirm the usefulness of this ratio and calculated a summative sensitivity of the ratio of $84 \%$ with a specificity of $78 \%$ to predict EV. The sensitivity of this ratio was also influenced by etiology of the liver disease with a sensitivity of $92 \%$ in viral liver cirrhosis [15]. Using the platelet count to spleen diameter ratio as previously described [7], the sensitivity was somewhat lower with our dataset. The reasons might be the greater variety of causes for liver disease in our cohort than in previous evaluations. Another non-invasive method is the use of computed tomography (CT) imaging with a sensitivity of $90 \%$ and a specificity of $72 \%$ for the detection of EV [16]. The higher sensitivity is traded against higher costs, exposure to irradiation, and the use of contrast agents. Other non-invasive measurements such as liver stiffness measurements are promising but further studies need to be performed. Meta-analysis of data so far collected by using transient elastography (FibroScan ${ }^{\circ}$ ) showed lower prognostic values for liver stiffness [17]. A meta-analysis of studies using different modes of elastography techniques to measure spleen stiffness showed heterogeneous results to detect EV [18]. The sensitivity of liver stiffness was $84 \%$ in predicting any varices, compared to $78 \%$ using the stiffness of the spleen as parameter. The specificity of the spleen stiffness was higher when compared to liver stiffness (76\% versus 62\%) [18] [16]. The use of capsule endoscopy to detect EV is also discussed in the literature [19] but high costs and its semi-invasive nature need to be kept in mind. Because of those limitations of the aforementioned non-invasive methods, the use of GBWT could represent a novel and feasible clinical marker for the detection of EV.

Table 4 Results of logistic regression analysis for prediction esophageal varices

\begin{tabular}{lllllll}
\hline \multicolumn{6}{l}{ Logistic regression for prediction esophageal varices } \\
\hline & $\mathrm{B}$ & S.E. & df & $p$ & $\mathrm{OR}=\operatorname{Exp}(\mathrm{B})$ & $95 \% \mathrm{Cl}$ for OR \\
\hline GBWT & -0.323 & 0.155 & 1 & 0.037 & 0.724 & $0.534-0.977$ \\
Spleen diameter & -0.023 & 0.009 & 1 & 0.007 & 0.977 & $0.961-0.994$ \\
Platelet count & 0.009 & 0.003 & 1 & 0.001 & 1.009 & $1.004-1.015$ \\
Ascites & 1.674 & 0.717 & 1 & 0.019 & 5.336 & $1.310-21.731$
\end{tabular}

$B$ Regression coefficient; S.E. Standard error; $d f$ Degree of freedom; OR Odds ratio; GBWT Gall bladder wall thickness
Alcantara previously published a cut-off value of $4.35 \mathrm{~mm}$ for a thickened gall bladder wall and found a sensitivity of $60 \%$ and a specificity of $90 \%$ regarding the presence of EV in pediatric patients [20]. The sensitivity was higher than in our study, although a higher value was used as cut-off. Other reasons for this difference might be the different patient cohorts, since de Alcantara based his study on data from children with various causes of cirrhosis such as biliary atresia and autoimmune hepatitis [20]. In our study we used data from adult patients with chronic liver disease and common causes for cirrhosis in Western Europe, but lack of histological confirmation in almost half of them. The cut-off-value of $4 \mathrm{~mm}$ was arbitrarily set for univariate analysis and seems reasonable since a lower value could be measured in individuals that did not fast overnight with a higher rate of falsely positive cases. A higher cut-off value would lower sensitivity.

In addition, a lowered velocity within the portal vein seems reasonable in the presence of portal hypertension and esophageal varices, but existing data are still conflicting. An Indian study of sonographic parameters predicting esophageal varices in 56 patients showed a significant difference of mean portal vein velocity. In presence of esophageal varices the mean velocity was $14.77 \mathrm{~cm} / \mathrm{s}$ and in absence of varices $17.66 \mathrm{~cm} / \mathrm{s}$ [21]. However, Li et al. did not report a significant difference in portal vein velocity between patients with and without esophageal varices. The mean velocity was $15.3 \mathrm{~cm} / \mathrm{s}$ in healthy individuals, $14.2 \mathrm{~cm} / \mathrm{s}$ in patients with first degree varices, $13.1 \mathrm{~cm} / \mathrm{s}$ in second degree varices and $12.0 \mathrm{~cm} / \mathrm{s}$ in third degree varices [22]. In our study there

Table 5 Sensitivity, specificity, positive and negative predictive value and area under the curve (AUC) for the presence of EV using the combination of platelet count/GBWT ratio (> 46.2), and platelet count/spleen diameter 909 as described by Giannini et al. [7]

\begin{tabular}{lll}
\hline Parameters & $\begin{array}{l}\text { Platelet count/GBWT } \\
(>46.2)\end{array}$ & Th/Spleen (<909) \\
\hline Sensitivity & $78 \%$ & $63 \%$ \\
Specificity & $86 \%$ & $87 \%$ \\
Positive predictive value & $76 \%$ & $75 \%$ \\
Negative predictive value & $87 \%$ & $80 \%$ \\
Area under the curve & $0.864(\mathrm{Cl} \mathrm{0.809-0.919)}$ & $0.841(\mathrm{Cl} \mathrm{0.782-0.901}$ \\
\hline
\end{tabular}


platelet count/GBWT: AUC 0.864 (Cl 0.809-0.919)

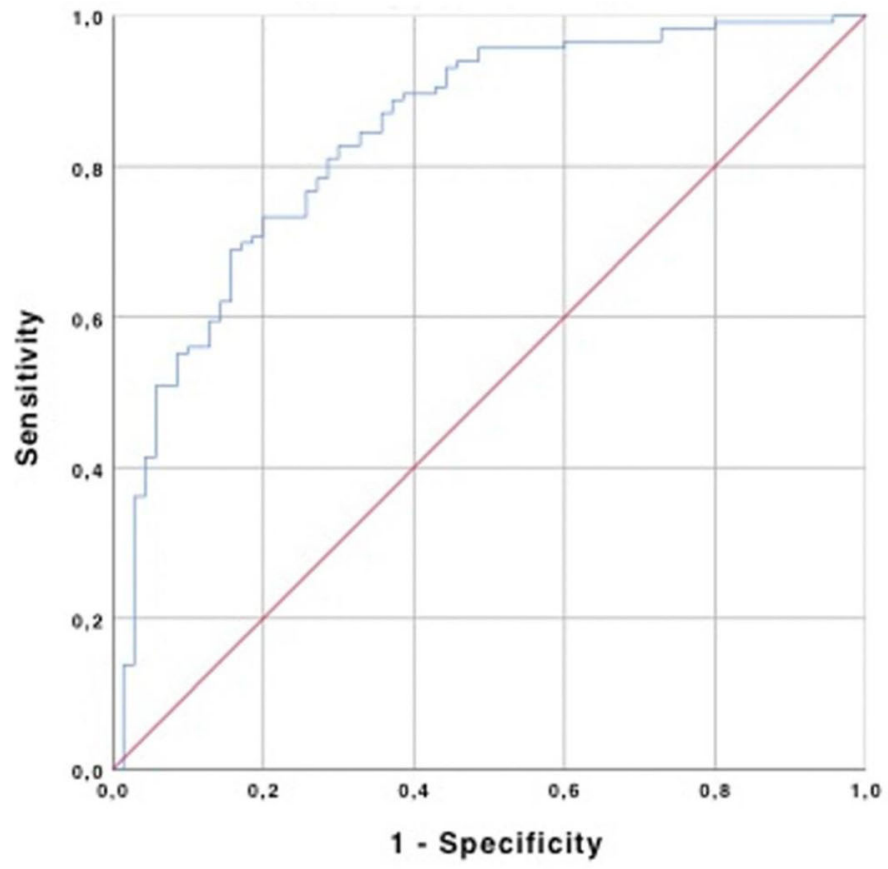

platelet count/spleen diameter: AUC 0.841 (Cl 0.782-0.901)

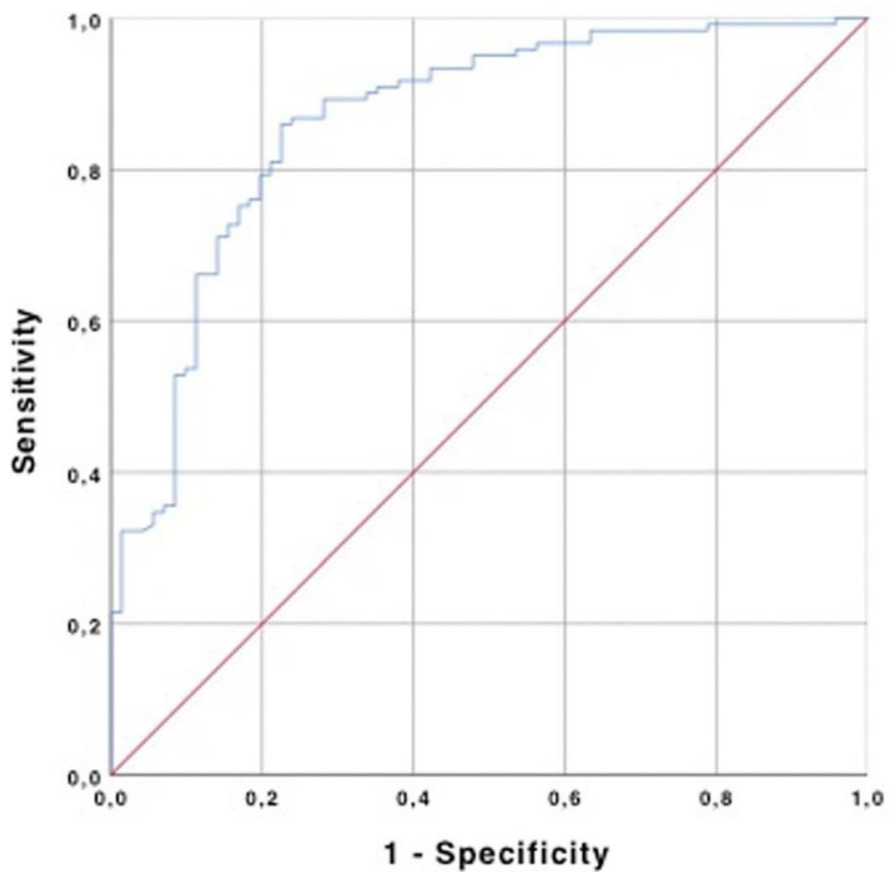

Fig. 3 Receiver operating characteristic (ROC) analysis and area under the curve (AUC) Top platelet count/GBWT ratio. Bottom platelet count/spleen diameter ratio

was no difference in average portal vein velocity with $18.0 \mathrm{~cm} / \mathrm{s}$ in both groups.

Our study has several limitations. First, ultrasonography and endoscopy were not always performed within a few days and may have biased results in case of rapidly changing endoscopy or ultrasonography findings. Furthermore, retrospective data collection could not establish a clear cause for chronic liver disease in almost $20 \%$ of patients. 
Secondly, although performed by experienced Gastroenterology trainees and consultants, GBWT measurements were performed by only one examiner, and inter-observer variability could thus not be accounted for. Third, we could not detect significant differences between small and large EV most likely due to the relatively low number of $3^{\circ} \mathrm{EVs}(n=9)$ in the EV cohort.

\section{Conclusions}

To conclude, GBWT may improve the non-invasive monitoring of liver disease patients to assess the risk for the presence of EV. However, a distinction between different severity grades of EV was not possible with the cut-off values used in our study. To improve upon the predictive value of GBWT, the combination with additional non-invasive parameters such as the platelet count is recommended.

\section{Abbreviations}

AUCa: Area under the curve; Cl: Confidence interval; EV: Esophageal varices; GBWT: Gall bladder wall thickening; INR: International normalized ratio; ROC: Receiver operating characteristic

\section{Availability of data and materials}

The datasets used and/or analyzed during the current study are available from the corresponding author on reasonable request.

\section{Authors' contributions \\ AN and SK initiated the study. BT and RM collected the data for this study. BT, AA and GP analyzed and interpreted the patient data. AN, SK and VE were major contributors in writing the manuscript. All authors read and approved the final manuscript.}

\section{Ethics approval and consent to participate}

The Ethics Committee at University Medical Centre Goettingen waived the need.

for written informed consent from the participants for this retrospective study and approved the study (Registration no. 24/7/15AN). This study also conformed to the Helsinki Declaration (2013) and local legislation.

\section{Consent for publication}

Not applicable.

\section{Competing interests}

The authors declare that they have no competing interests.

\section{Publisher's Note}

Springer Nature remains neutral with regard to jurisdictional claims in published maps and institutional affiliations.

Received: 28 January 2018 Accepted: 25 July 2018

Published online: 02 August 2018

References

1. Blachier M, Leleu H, Peck-Radosavljevic M, Valla D-C, Roudot-Thoraval F. The burden of liver disease in Europe: a review of available epidemiological data. J Hepatol. 2013;58:593-608.

2. Fleming KM, Aithal GP, Card TR, West J. All-cause mortality in people with cirrhosis compared with the general population: a population-based cohort study. Liver Int Off J Int Assoc Study Liver. 2012:32:79-84.

3. Merli M, Nicolini G, Angeloni S, Rinaldi V, De Santis A, Merkel C, et al. Incidence and natural history of small esophageal varices in cirrhotic patients. J Hepatol. 2003;38:266-72.
4. Hsieh Y-C, Lee K-C, Chen P-H, Su C-W, Hou M-C, Lin H-C. Acute kidney injury predicts mortality in cirrhotic patients with gastric variceal bleeding. Gastroenterol Hepatol. 2017;32:1859-66

5. de Franchis $R$, Primignani M. Natural history of portal hypertension in patients with cirrhosis. Clin Liver Dis. 2001;5:645-63.

6. Way LW, Pellegrini CA, editors. Surgery of the gallbladder and bile ducts. Philadelphia: Saunders; 1987.

7. Giannini E, Botta F, Borro P, Risso D, Romagnoli P, Fasoli A, et al. Platelet count/spleen diameter ratio: proposal and validation of a non-invasive parameter to predict the presence of oesophageal varices in patients with liver cirrhosis. Gut 2003:52:1200-5.

8. Pagliaro, et al. In: Bosch J, Groszmann RJ, editors. Portal Hypertens Pathophysiol Treat Portal hypertension in cirrhosis: natural history. Oxford: Blackwell Science; 1992. p. 72-92.

9. Denzer U, Beilenhoff U, Eickhoff A, Faiss S, Hüttl P, In der Smitten S, et al. S2k guideline: quality requirements for gastrointestinal endoscopy, AWMF registry no. 021-022. Z Gastroenterol. 2015:53:E1-227.

10. Sarin SK, Lahoti D, Saxena SP, Murthy NS, Makwana UK. Prevalence, classification and natural history of gastric varices: a long-term follow-up study in 568 portal hypertension patients. Hepatol Baltim Md. 1992;16:1343-9.

11. Maruyama H, Kobayashi K, Kiyono S, Ogasawara S, Ooka Y, Suzuki E, et al. Left gastric vein-based noninvasive test for esophageal varices: a same-day comparison of portal hemodynamic assessment with endoscopic appearance. Clin Transl Gastroenterol. 2018:9:154.

12. Li C, Yang Z, Ma E, Liu Y. Analysis of the correlation between the degree of GBWT and hemodynamic changes of portal vein system. Sheng Wu Yi Xue Gong Cheng Xue Za Zhi J Biomed Eng Shengwu Yixue Gongchengxue Zazhi. 2010;27:583-5. 625

13. Colli A, Cocciolo M, Buccino G, Parravicini R, Martinez E, Rinaldi G, et al. Thickening of the gallbladder wall in ascites. J Clin Ultrasound. 1991;19:357-9.

14. Giannini E, Botta F, Borro P, Malfatti F, Fumagalli A, Testa E, et al. Relationship between thrombopoietin serum levels and liver function in patients with chronic liver disease related to hepatitis $C$ virus infection. Am J Gastroenterol. 2003;98:2516-20

15. Chen R, Deng H, Ding X, Xie C, Wang W, Shen Q. Platelet count to spleen diameter ratio for the diagnosis of gastroesophageal varices in liver cirrhosis: a systematic review and meta-analysis. Gastroenterol Res Pract. 2017;2017:7407506.

16. Tseng Y-J, Zeng X-Q, Chen J, Li N, Xu P-J, Chen S-Y. Computed tomography in evaluating gastroesophageal varices in patients with portal hypertension: a meta-analysis. Dig Liver Dis Off J Ital Soc Gastroenterol Ital Assoc Study Liver. 2016:48:695-702

17. Pu K, Shi J-H, Wang X, Tang Q, Wang X-J, Tang K-L, et al. Diagnostic accuracy of transient elastography (FibroScan) in detection of esophageal varices in patients with cirrhosis: a meta-analysis. World J Gastroenterol. 2017:23:345-56

18. Singh S, Eaton JE, Murad MH, Tanaka H, lijima H, Talwalkar JA. Accuracy of spleen stiffness measurement in detection of esophageal varices in patients with chronic liver disease: systematic review and meta-analysis. Clin Gastroenterol Hepatol Off Clin Pract J Am Gastroenterol Assoc. 2014;12:935-945.e4.

19. Colli A, Gana JC, Turner D, Yap J, Adams-Webber T, Ling SC, et al. Capsule endoscopy for the diagnosis of oesophageal varices in people with chronic liver disease or portal vein thrombosis. Cochrane Database Syst Rev. 2014;10:CD008760

20. de Alcantara RV, Yamada RM, Cardoso SR, de Fátima M, Servidoni CP, Hessel G. Ultrasonographic predictors of esophageal varices. J Pediatr Gastroenterol Nutr. 2013:57:700-3.

21. Chakrabarti R, Sen D, Khanna V. Is non-invasive diagnosis of esophageal varices in patients with compensated hepatic cirrhosis possible by duplex Doppler ultrasonography? Indian J Gastroenterol Off J Indian Soc Gastroenterol. 2016:35:60-6.

22. Li F-H, Hao J, Xia J-G, Li H-L, Fang H. Hemodynamic analysis of esophageal varices in patients with liver cirrhosis using color Doppler ultrasound. World Gastroenterol. 2005:11:4560-5. 